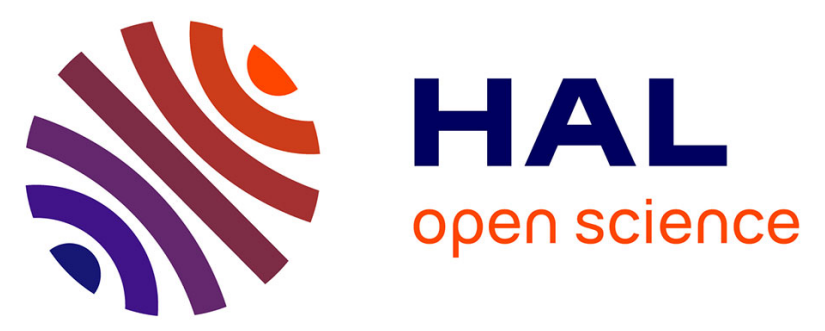

\title{
EMT Transcription Factor ZEB1 Represses the Mutagenic POL $\theta$-Mediated End-Joining Pathway in Breast Cancers
}

Mélanie K Prodhomme, Roxane M Pommier, Camille Franchet, Frédérique Fauvet, Valérie Bergoglio, Pierre Brousset, Anne-Pierre Morel, Anne-Cécile Brunac, Mojgan Devouassoux-Shisheboran, Virginie Petrilli, et al.

\section{To cite this version:}

Mélanie K Prodhomme, Roxane M Pommier, Camille Franchet, Frédérique Fauvet, Valérie Bergoglio, et al.. EMT Transcription Factor ZEB1 Represses the Mutagenic POL $\theta$-Mediated End-Joining Pathway in Breast Cancers. Cancer Research, 2021, 81 (6), pp.1595 - 1606. 10.1158/0008-5472.can-202626. hal-03407927

\section{HAL Id: hal-03407927 https://hal.science/hal-03407927}

Submitted on 29 Oct 2021

HAL is a multi-disciplinary open access archive for the deposit and dissemination of scientific research documents, whether they are published or not. The documents may come from teaching and research institutions in France or abroad, or from public or private research centers.
L'archive ouverte pluridisciplinaire $\mathbf{H A L}$, est destinée au dépôt et à la diffusion de documents scientifiques de niveau recherche, publiés ou non, émanant des établissements d'enseignement et de recherche français ou étrangers, des laboratoires publics ou privés. 


\section{EMT Transcription Factor ZEB1 Represses the Mutagenic

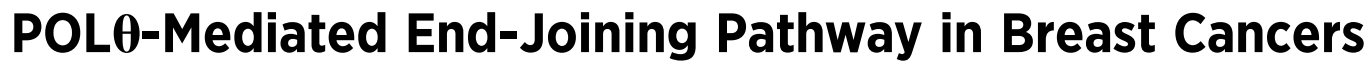

Mélanie K. Prodhomme ${ }^{1,2}$, Roxane M. Pommier ${ }^{1,2,3}$, Camille Franchet ${ }^{4}$, Frédérique Fauvet ${ }^{1,2}$, Valérie Bergoglio ${ }^{5}$, Pierre Brousset ${ }^{4}$, Anne-Pierre Morel ${ }^{1,2}$, Anne-Cécile Brunac ${ }^{4}$, Mojgan Devouassoux-Shisheboran ${ }^{1,2}$, Virginie Petrilli ${ }^{6}$, Caroline Moyret-Lalle ${ }^{1,2}$, Jean-Sébastien Hoffmann ${ }^{4}$, Alain Puisieux ${ }^{1,2,7}$, and Agnès Tissier ${ }^{1,2}$

\section{ABSTRACT}

A characteristic of cancer development is the acquisition of genomic instability, which results from the inaccurate repair of DNA damage. Among double-strand break repair mechanisms induced by oncogenic stress, the highly mutagenic thetamediated end-joining (TMEJ) pathway, which requires DNA polymerase theta $(\mathrm{POL} \theta)$ encoded by the $P O L Q$ gene, has been shown to be overexpressed in several human cancers. However, little is known regarding the regulatory mechanisms of TMEJ and the consequence of its dysregulation. In this study, we combined a bioinformatics approach exploring both Molecular Taxonomy of Breast Cancer International Consortium and The Cancer Genome Atlas databases with CRISPR/Cas9-mediated depletion of the zinc finger E-box binding homeobox 1 (ZEB1) in claudin-low tumor cells or forced expression of ZEB1 in basal-like tumor cells, two triple-negative breast cancer (TNBC) subtypes, to demonstrate that ZEB1 represses

\section{Introduction}

Chromosomal instability (CIN) is a hallmark of cancer (1), arising notably through the error-prone repair of double-strand breaks (DSB), ultimately resulting from oncogenic activation (2). Indeed, the usage of unfaithful pathways eventually leads to inappropriate endjoining events at the origin of genomic instability (3). Alongside the well-documented homologous recombination (HR) and canonical nonhomologous end-joining (c-NHEJ) DSB repair pathways $(4,5)$, mammalian cells also rely on an independent highly mutagenic

'Université de Lyon, Université Claude Bernard Lyon 1, INSERM 1052, CNRS 5286, Centre Léon Bérard, Cancer Research Centre of Lyon, Équipe Labellisée Ligue contre le Cancer, Lyon, France. ${ }^{2}$ LabEx DEVweCAN, Université de Lyon, Lyon, France. ${ }^{3}$ Gilles Thomas Bioinformatics Platform, Centre Léon Bérard, Cancer Research Centre of Lyon, Lyon, France. ${ }^{4}$ Laboratoire de Pathologie, Laboratoire d'excellence Toulouse Cancer, Institut Universitaire du Cancer-Toulouse, Oncopole, Toulouse Cedex, France. ${ }^{5}$ Cancer Research Centre of Toulouse, INSERM UMR 1037, Toulouse, France. ${ }^{6}$ Université de Lyon, Université Claude Bernard Lyon 1, INSERM 1052, CNRS 5286, Centre Léon Bérard, Cancer Research Centre of Lyon, Lyon, France. ${ }^{7}$ Institut Curie, PSL Research University, Paris, France.

Note: Supplementary data for this article are available at Cancer Research Online (http://cancerres.aacrjournals.org/).

A. Puisieux and A. Tissier contributed equally as co-supervisors of this article.

Corresponding Authors: Agnès Tissier, Cancer Research Centre of Lyon, Lyon 69373, France. Phone: 33-426-556-791; E-mail: agnes.tissier@inserm.fr; and Alain Puisieux, Institut Curie 26 rue d’UIm 75248 Paris Cedex 05 France.

E-mail: alain.puisieux@curie.fr

Cancer Res 2021;81:1595-606

doi: 10.1158/0008-5472.CAN-20-2626

(c)2020 American Association for Cancer Research.
POLQ expression. ZEB1, a master epithelial-to-mesenchymal transition-inducing transcription factor, interacted directly with the POLQ promoter. Moreover, downregulation of POLQ by ZEB1 fostered micronuclei formation in TNBC tumor cell lines. Consequently, ZEB1 expression prevented TMEJ activity, with a major impact on genome integrity. In conclusion, we showed that ZEB1 directly inhibits the expression of POLQ and, therefore, TMEJ activity, controlling both stability and integrity of breast cancer cell genomes.

Significance: These findings uncover an original mechanism of TMEJ regulation, highlighting ZEB1 as a key player in genome stability during cancer progression via its repression of $P O L Q$.

See related commentary by Carvajal-Maldonado and Wood, p. 1441

theta-mediated end-joining (TMEJ) pathway, corresponding to one of the initial alternative end-joining pathways defined as a $\mathrm{Ku}$ independent c-NHEJ, and then renamed microhomology-mediated end joining on the basis of the mechanism or TMEJ, on the basis of the key actor (6-9). In TMEJ, DSBs are sealed by microhomologymediated base pairing of DNA single strands, yielding products systematically associated with short DNA deletions and insertions, potentially generating chromosomal translocations and mutagenic rearrangements (10-13). Key TMEJ actors in human cells include the A-family DNA polymerase theta (POL $\theta$ ) encoded by the $P O L Q$ gene, PARP1 (14-16), and DNA ligase III $\alpha$ (encoded by the LIG3 gene; refs. 7, 17). Thus far, the mechanisms regulating TMEJ in normal and cancer cells are totally unknown. In this work, we gain insight into the regulatory process of triple-negative breast cancers (TNBC). TNBCs are aggressive breast malignancies that are characterized by the lack of estrogen and progesterone receptor expression and the absence of HER2 overexpression. TNBCs represent up to $20 \%-25 \%$ of all breast carcinomas. According to molecular classifications based on gene expression profiles, TNBCs are essentially composed of basallike and claudin-low subtypes (18). Claudin-low tumors display a low level of expression of cell-cell adhesion molecules, such as claudins or E-cadherin, encoded by the $\mathrm{CDH} 1$ gene. In addition, they are highly enriched in mesenchymal traits and stem cell features, and are therefore, considered to be the most primitive breast cancers with poor survival outcomes compared with many other breast cancer subtypes $(19,20)$.

ZEB1, a transcription factor inducer of the epithelial-tomesenchymal transition (EMT), modulates breast cancer cell plasticity by conferring stemness properties to the cells via numerous mechanisms, including the transcriptional repression of epithelial actors, such as E-cadherin (CDH1 gene; ref. 21), or miRNAs like miR-200 (22). 
ZEB1 expression was shown to promote malignant transformation while maintaining genome stability (23). In particular, high ZEB1 expression is causally associated with claudin-low tumors, characterized by a subnormal genomic landscape (24). ZEB1 has been implicated both in preventing the formation of oncogene-induced DNA damage and in increasing the clearance of DNA breaks. On one hand, ZEB1 is able to protect mammary stem cells against oncogene-induced damage through the activation of a preemptive antioxidant program, and favors tumorigenesis in the absence of gross genomic instability (24). On the other hand, the kinase ATM, critical player in DNA damage response, phosphorylates and stabilizes ZEB1, triggering the cell-cycle checkpoint CHK1 stabilization at the origin of treatment resistance (25). Owing to these pleiotropic effects, ZEB1 is considered to be the central factor in providing cancer cells with a high level of plasticity and may thus, be pivotal in the development of therapeutically resistant cancers.

Here, we show that ZEB1 directly controls the expression of POLQ and influences not only genome stability of breast cancer cells, but also genome integrity.

\section{Materials and Methods}

Bioinformatics and statistical analyses of public databases (The Cancer Genome Atlas and Molecular Taxonomy of Breast Cancer International Consortium)

All bioinformatics and statistical analyses were carried out with the R software (version 3.5.1; ref. 26). Figures were created using either the R software or GraphPad Prism 6.0 (GraphPad Software Inc.; RRID:SCR_002798). OncoPrint plots were generated using the ComplexHeatMap open source software (https:/github.com/jokergoo/ ComplexHeatmap; RRID:SCR_017270; ref. 27).

\section{Expression data processing}

Molecular Taxonomy of Breast Cancer International Consortium (METABRIC) microarray expression data from discovery and validation sets were extracted from the EMBL-EBI archive (EGA; http://www.ebi.ac.uk/ega/; accession no. EGAS00000000083; RRID: SCR_ 004944; "normalized expression data" files; ref. 28). The expression levels of different probes associated with the same Entrez Gene ID were averaged for each sample to obtain a single expression value by gene.

The Cancer Genome Atlas (TCGA; RRID:SCR_003193) breast invasive carcinoma (BRCA) RNA sequencing expression data were extracted as fragments per kilobase of transcript per million mapped reads (FPKM) values from the Genomic Data Commons (GDC) data portal (https://portal.gdc.cancer.gov/). FPKM data by gene were converted to transcripts per kilobase million (TPM) as follows: for each gene, $g \in \mathrm{G}$ and each sample, $\mathrm{s} \in \mathrm{S}$,

$$
\operatorname{TPM}(g, s)=\left(\frac{\operatorname{FPKM}(g, s)}{\sum_{i=1}^{G} \operatorname{FPKM}(i, s)}\right) \times 10^{6}
$$

Expression data by gene from TCGA and METABRIC (discovery and validation sets independently) were finally merged in a common file, keeping all genes present in both datasets, and batch normalization was performed using the R function ComBat from sva package $(29,30)$. The final expression dataset was composed of 18,845 genes and 3,083 breast tumor samples.

\section{Copy-number data processing}

METABRIC segmented copy-number data from discovery and validation sets were extracted from the EMBL-EBI archive [EGA, http:// www.ebi.ac.uk/ega/; accession no. EGAS00000000083; RRID:SCR 004944; "segmented (CBS) copy-number aberrations" files; ref. 28].

TCGA (RRID:SCR_003193) BRCA segmented copy-number data were extracted from the GDC data portal repository (files corresponding to alignments on the hg19 version of the human genome without germline copy-number variation were chosen).

As described previously $(24,31)$, fraction of genomic alterations (FGA) was evaluated from TCGA and METABRIC segmented copynumber data (both generated from Affymetrix SNP6.0 arrays) as follows:

$$
F G A=\frac{\sum_{C N i>W M+T} L(i)}{\left(\sum L(i)\right)}+\frac{\sum_{C N i<W M-T} L(i)}{\left(\sum L(i)\right)}
$$

For each segment $i, \mathrm{CN} i$ is the mean $\log \mathrm{R}$ ratio along segment $i, L(i)$ is the length of segment $i$, WM is the weighted median of CNiby $L(i)$ for each sample $I$, and $T$ is the threshold value of the $\mathrm{CN} i$ above which the segments are considered to be altered. In other words, FGA is the ratio of the sum of the lengths of all segments with signal above the threshold to the sum of all segment lengths, that is, FGA is the percentage of the genome displaying an aberrant copy number (deletion and amplification). For METABRIC and TCGA TNBCs analysis, $T$ was set as 0.1 , taking into account that TNBCs were not sorted by cellularity.

\section{Triple negative status}

Estrogen, progesterone, and HER2 receptors' statuses were determined through expression analysis of the ESR1, PGR, and ERBB2 genes, respectively. Using global distribution of each gene expression, samples were classified into positive and negative subgroups using mclust $\mathrm{R}$ package (version 5.4.2), which decomposes the global distribution into Gaussian mixture models to classify samples (32).

\section{Breast cancer subtype assignment}

Breast cancer molecular subtype attribution [basal-like, luminal A, luminal B, Her2, normal-like, and integrative clusters (IntClust)] was performed using the R package "genefu," version 3.8 (33). Basal-like, luminal A, luminal B, Her2, and normal-like subtype assignments were computed with five different algorithms (PAM50, AIMS, SCMGENE, SSP2006, and SCMOD2; refs. 34-38). An assignment was considered final if defined by at least three different algorithms. In case of divergence between classifiers, PAM50 subtype attribution was conserved.

The claudin-low subtype classification was defined by nearest centroid method. To achieve this, we computed the Euclidean distance between each sample and the previously described claudin-low and non-claudin-low centroids, using the 1,667 genes defined by Prat and colleagues as significantly differentially expressed between claudin-low tumors and all other molecular subtypes (20).

\section{Cell cultures}

HMEC-hTERT was generated in the laboratory as described previously (23). HMEC-hTERT was cultured in 1:1 DMEM/Ham F12 medium with 1\% GlutaMAX (Gibco-Thermo Fisher Scientific; catalog no. 31331093) supplemented with $10 \mathrm{ng} / \mathrm{mL}$ Human EGF (PromoCell; catalog no. C-60170), $0.5 \mathrm{mg} / \mathrm{mL}$ Hydrocortisone (Sigma-Aldrich; catalog no. H0888), and $10 \mathrm{mg} / \mathrm{mL}$ insulin (Actrapid, Novonordisk).

BT-20 (human mammary carcinoma cells, ATCC) was maintained in minimum essential medium with $1 \%$ GlutaMAX (Gibco-Thermo Fisher Scientific; catalog no. 41090093). MDA-MB-468 (human mammary adenocarcinoma cells, derived from metastatic site: pleural effusion, DSMZ) was maintained in Leibovitz L15 with $1 \%$ GlutaMAX (Gibco-Thermo Fisher Scientific; catalog no. 31415086). HCC70 
(human mammary primary ductal carcinoma, ATCC) was cultured in RPMI1640 with 1\% GlutaMAX (Gibco-Thermo Fisher Scientific; catalog no. 61870044) supplemented with $1.5 \mathrm{~g} / \mathrm{L}$ sodium bicarbonate (Gibco-Thermo Fisher Scientific; catalog no. 25080060), $10 \mathrm{mmol} / \mathrm{L}$ HEPES (Gibco-Thermo Fisher Scientific; catalog no. 15630056), and $1 \mathrm{mmol} / \mathrm{L}$ sodium pyruvate (Gibco-Thermo Fisher Scientific; catalog no. 11360039). HCC1937 (human mammary carcinoma cells, ATCC) and BT-549 (human mammary carcinoma cells, ATCC) were cultured in RPMI1640 with 1\% GlutaMAX (Gibco-Thermo Fisher Scientific; catalog no. 61870044). SUM159 cells were a gift from Hasan Korkaya's laboratory at Augusta University (Augusta, GA). SUM159 was cultured in Ham-F12 with glutamine (Gibco-Thermo Fisher Scientific; catalog no. 21765037) supplemented with $3.2 \mu \mathrm{g} / \mathrm{mL}$ Gentamicin (Gibco-Thermo Fisher Scientific; catalog no. 15710049), $5 \mathrm{mg} / \mathrm{mL}$ insulin (Actrapid, Novonordisk), and $2 \mathrm{mg} / \mathrm{mL}$ hydrocortisone (Sigma-Aldrich; catalog no. H0888). MDA-MB-231 (human mammary adenocarcinoma cells, ATCC), Hs 578T (human mammary carcinoma cells, ATCC), CAL-120 (human mammary adenocarcinoma cells, DSMZ), Phoenix, Plat-E, and HEK293T were maintained in DMEM with 1\% GlutaMAX (Gibco-Thermo Fisher Scientific; catalog no. 31966047).

All media were supplemented with $10 \%$ FCS (Sigma-Aldrich or Eurobio) and penicillin-streptomycin $(100 \mu \mathrm{g} / \mathrm{mL}, 100 \mathrm{U} / \mathrm{mL}$, respectively, Gibco-Thermo Fisher Scientific; catalog no. 15140130), except for MDA-MB-468, which was supplemented with $20 \%$ FCS (SigmaAldrich)

All cell lines were kept at $37^{\circ} \mathrm{C}$ in a $5 \% \mathrm{CO}_{2} / 95 \%$ air incubator, and were routinely tested negative for Mycoplasma contamination using the Lonza MycoAlert PLUS Mycoplasma Detection Kit (Lonza; catalog no. LT07-318).

\section{Lentiviral and retroviral infections}

To produce lentiviral particles, $2 \times 10^{6}$ HEK293T cells were transfected using the GeneJuice Transfection Reagent (Sigma-Aldrich; catalog no. 70967-4), according to the manufacturer's instructions, with $13 \mu \mathrm{g}$ of total lentiviral expression vectors $(5.1 \mu \mathrm{g}$ pCMVdeltaR8.91, $1.3 \mu \mathrm{g}$ phCMVG-VSVG, and $6.6 \mu \mathrm{g}$ plasmid of interest). The pCMVdeltaR8.91 and phCMVG-VSVG vectors were gifts from D. Nègre (International Centre for Infectiology Research, INSERM U1111-CNRS UMR5308-ENS de Lyon-UCB Lyon1, EVIR Team, Lyon, France).

To produce retroviral particles, $2 \times 10^{6}$ Phoenix cells were transfected by GeneJuice Transfection Reagent (Sigma-Aldrich; catalog no. 70967-4), according to the manufacturer's instructions, with $10 \mu \mathrm{g}$ of plasmid of interest.

For both infections, the supernatant was collected, filtered, supplemented with 5 or $10 \mu \mathrm{g} / \mathrm{mL}$ Polybrene (Sigma-Aldrich; catalog no. H9268) 48 hours after transfection and combined with the targeted cells for 12 hours.

The ZEB1-depletion model in MDA-MB-231 (MDA-MB-231 $\mathrm{ZEB1}^{-/-}$clones) using the CRISPR-cas9 gene editing technology was generated. Scrambled sgRNA/Cas9 All-in-One Lentivector (Applied Biological Materials; catalog no. K010) and ZEB1 sgRNA/Cas9 All-inOne Lentivector (Human) (target 1: 5'-CACCTGAAGAGGACCAG$3^{\prime}$; Applied Biological Materials; catalog no. K2671006) lentiviral particles were used to infect MDA-MB-231 cells. Scrambled sgRNA/Cas9 and ZEB1 sgRNA/Cas9 cells were selected with Puromycin (InvivoGen; catalog no. ant-pr-1) at $1 \mu \mathrm{g} / \mathrm{mL} 48$ hours after infection. After cloning by limiting dilution, single cells were grown for approximately 3 weeks and colonies were screened for knockouts by quantitative PCR and genomic DNA sequencing and Western blot- ting. Genomic DNA sequencing was performed using the Sanger sequencing method with the following primers for amplification and sequencing: $5^{\prime}$-TGAACTGAACGTCAGAGTGGT-3' (forward) and 5'-TCACGTGCAGTGGCATTACT-3' (reverse).

To generate the model with forced overexpression of ZEB1 in noneZEB1-expressing cells, BT-20 and HCC70 basal-like cells were infected with retroviral pBabe expression vectors containing $Z E B 1$. Cells were selected with neomycin at $100 \mu \mathrm{g} / \mathrm{mL}$ for BT-20 (GibcoThermo Fisher Scientific; catalog no. 10131027) and with puromycin (InvivoGen; catalog no. ant-pr-1) at $1 \mu \mathrm{g} / \mathrm{mL} 48$ hours after infection.

For luciferase assay, MDA-MB-231 cells were infected with four lentiviral reporter pEZX-LvPG04 plasmids (GeneCopoeia). Two $P O L Q$ promoter constructs, a $\mathrm{CDH} 1$ promoter (GeneCopoeia; catalog no. HPRM45458-LvPG04) and a negative promoter (GeneCopoeia; catalog no. NEG-LvPG04) as a control, were independently transduced. The POLQ promoter -691 bp was generated from the POLQ promoter $-1,280$ bp (GeneCopoeia; catalog no. HPRM54321LvPG04-01) digested by EcoR1 and Spe1 restriction enzymes and reconstituted (New England Biolabs).

To generate the model with forced overexpression of $P O L Q$ in MDA-MB-231, lentiviral PCDH-EF1-FHC-POLQ vector containing human POLQ cDNA (Addgene; catalog no. 64875 for POLQ and catalog no. 64874 for empty control) was used. Cells were selected with Puromycin (InvivoGen; catalog no. ant-pr-1) at $1 \mu \mathrm{g} / \mathrm{mL} 48$ hours after infection.

\section{Drugs and siRNA}

6-Thioguanine (6-TG; Sigma-Aldrich; catalog no. A4882) stock solution was dissolved in $\mathrm{NaOH} 0.1 \mathrm{~N}$ and cytochalasin $\mathrm{B}$ (Sigma-Aldirch; catalog no. C6762-1MG) stock solution was dissolved in DMSO.

Transient siRNA-mediated knockdown was performed with INTERFERin Reagent (PolyPlus-transfection; Ozyme; catalog no. POL409-50) according to the manufacturer's protocol during the time of the experiment (kinetic or single point). siRNAs were used at a final concentration of $2 \mathrm{nmol} / \mathrm{L}$ for MDA-MB-231 or $8 \mathrm{nmol} / \mathrm{L}$ for BT-549 and SUM159, and cells were treated every day. siRNA sequences (Eurogentec) are as follows: siRNA nontargeted siNT, 5'-GGUUUGGCUGGGGUGUUAU-3'; siZEB1\#1, 5'-GGUAGAUGGUAAUGUAAUA- $3^{\prime}$; siZEB1\#2, $5^{\prime}$-GCAACAGGGAGAAUUAUUATT-3'; and $S I P O L Q, 5^{\prime}$-CAAACAACCCUUAUCGUAAA-3'.

\section{Western blot analysis and antibodies}

Equal amounts of protein of each sample were analyzed using SDS-PAGE, electrophoretic transfer, immunoblotting, and chemiluminescence detection. Briefly, cells were washed and scratched with ice-cold PBS (Eurobio; catalog no. CS1PBS01K-BP) supplemented with Protease Inhibitor Cocktail (PIC, Sigma-Aldrich, catalog no. 11836145001), Phenyl-methane Sulfonyl Fluoride (PMSF, Sigma-Aldrich; catalog no. 93482), and Phosphatase Inhibitor Cocktail (PhoIC 2 and 3, Sigma-Aldrich; catalog no. P5726$5 \mathrm{ML}$ and catalog no. P0044-5ML, respectively) on ice. The cell pellets were lysed in $2 \%$ SDS, $125 \mathrm{mmol} / \mathrm{L}$ Tris, $\mathrm{pH}$ 6.8, PIC, PMSF, and PhoIC 2 and 3 on ice. After sonication, proteins were separated by SDS-PAGE and transferred to Polyvinylidene Difluoride Membranes (Bio-Rad; catalog no.1620177). Antibodies and dilutions were as follows: anti-ZEB1, 1:1,000 (polyclonal; Sigma Life Science; catalog no. HPA027524); anti-POL $\theta, 1: 10,000$ (as described previously; ref. 39); anti-PARP1, 1:1,000 [polyclonal PARP-1 (H-250); Santa Cruz Biotechnology; catalog no. sc-7150]; anti-LIG3, 1:1,000 (clone 6G9; Santa Cruz Biotechnology; catalog no. sc-56089); antiCAS9, 1:1,000 (clone 7A9-3A3; Cell Signaling Technology; catalog 
no. 14697); and anti-Human DNA Topoisomerase I, 1:1,000 (clone C-21; Cell Signaling Technology; catalog no. 556597). Speciesspecific secondary horseradish peroxidase (HRP)-coupled antibodies (Santa Cruz Biotechnology; goat anti-mouse catalog no. SC 2005 and mouse anti-rabbit catalog no. SC 2357) were used. Protein bands were visualized using Clarity or Clarity-max (Bio-Rad; catalog no. 1705061 and catalog no. 1705062, respectively) and the ChemiDoc MP System (Bio-Rad).

\section{Quantitative real-time PCR}

Total RNA was extracted with the RNeasy Mini Kit (Qiagen; catalog no. 74106) according to the manufacturer's recommendations. Reverse transcription was performed from $1 \mu \mathrm{g}$ total RNA with the Dynamo cDNA Synthesis Kit (Thermo Fisher Scientific; catalog no. F470L). The reverse transcription product was diluted 1:10 and used as cDNA template for qPCR analysis. TaqMan Quantitative PCR (Bioline Meridian Bioscience Europe; catalog no. BIO-86050) was used in the detection of PCR products in real-time in a CFX96 Real-time PCR Detection System (Bio-Rad) according to the manufacturer's instructions. Quantitative reverse-transcription PCR (qRT-PCR) was performed using $200 \mathrm{nmol} / \mathrm{L}$ of specific primers and DNA probes (Table 1; design on universal probe library by Roche Life Science). Conditions for the TaqMan method were 2 minutes at $50^{\circ} \mathrm{C}, 20$ seconds at $95^{\circ} \mathrm{C}$, and then 40 cycles, each consisting of 3 seconds at $95^{\circ} \mathrm{C}$ and 30 seconds at $60^{\circ} \mathrm{C}$. The housekeeping genes used were $U B B$ and glyceraldehyde3-phosphate dehydrogenase (GAPDH). The comparative $C_{\mathrm{t}}$ method was used to quantify the expression of the gene of interest. Refer to the list of primers and probes in Table 1.

\section{Human tumor samples and IHC analyses}

Ten rare positive and 22 negative previously characterized ZEB1 tumors (24) were analyzed for POLQ expression by RNAscope. RNA ISH for Hs-POLQ mRNA was performed on the Ventana Discovery Ultra Automated Slide Staining System (Roche Diagnostics) using RNAscope VS Universal HRP Reagent Kit (Brown; Advanced Cell Diagnostics, Inc.; catalog no. 323220) and RNAscope probe specific to the region of the gene encoding Homo sapiens DNA polymerase $\theta$ mRNA (catalog no. 465519) according to the manufacturer's instructions. Briefly, $5 \mu \mathrm{m}$ formalin-fixed, paraffin-embedded tissue sections were pretreated at $96^{\circ} \mathrm{C}$ for 16 minutes prior to hybridization with the target probes. Preamplifier, amplifier, and HRP-labeled oligos were then hybridized sequentially, followed by chromogenic precipitate development. RNA integrity was controlled by the use of a RNAscope probe specific to Hs-PPIB RNA (catalog no. 313909). A negative control with a probe specific to bacterial dapB RNA was also performed (catalog no. 312039). Specific RNA staining signal was identified as brown dots. Slides were digitized using Panoramic 250 Flash II Slide Scanner (3DHISTECH) with $40 \times$ objective (resolution, 0.12154 $\mu \mathrm{m} / \mathrm{pixel}$ ) and extended focus algorithm. For each case, three representative images were acquired at $\times 60$ magnification with CaseViewer (3DHISTECH). In the images, positive cells (cells with at least one
POLQ RNAscope signal in the nucleus) were manually annotated using dedicated annotation layers with the counter tool of the viewer Aperio ImageScope version 12.3.2 (Leica Biosystems). Percentage of stained cells, mean number of POLQ RNAscope signal per stained cell, and $\mathrm{H}$-score (product of the two previous scores) were calculated for each case. POLQ expression was considered low or high with respect to a threshold for $\mathrm{H}$-score at 30. This method for RNAscope quantification relies on an excellent interobserver correlation $\left(R^{2}=\right.$ $0.96 ; P<0.0001$, between two independent pathologists).

\section{Chromatin immunoprecipitation assay}

Chromatin immunoprecipitation (ChIP)-IT High Sensitivity Kit (Active Motif; catalog no. 53040) was used to determine the association of the transcription factor ZEB1 with POLQ- or CDH1-specific genomic regions. MDA-MB-231 and MDA-MB-231 ZEB1 ${ }^{-/-}$cells were subjected to cell fixation, $1 \%$ Formaldehyde (Sigma-Aldrich, catalog no. 252549-1L) on ice to cross-link the proteins bound to chromatin DNA. After washing, chromatin DNA was sheared by sonication to produce DNA fragments of around 200-1,000 bp. The same amounts of sheared DNA were used for immunoprecipitation using anti-ZEB1 antibody (GeneTex; catalog no. GTF105278) or an equal amount of pre-immune Rabbit IgG (Bio-Rad; catalog no. PRABP01, $10 \mathrm{mg}$ ). The immunoprecipitate was then incubated with protein $\mathrm{G}$ magnetic beads, and the antibody-protein $\mathrm{G}$ magnetic beads complex was collected for subsequent reverse cross-linking. The same amount of sheared DNA without antibody precipitation was processed for reverse cross-linking and served as an input control. DNA recovered from reverse cross-linking was used for qPCR to determine the abundance of the target DNA sequence(s) relative to the input chromatin. ChIP-qPCR primers for the POLQ promoter were: primers \#1: $5^{\prime}$-ACGTTCAGAACTCGTTCGCT-3' (forward) and $5^{\prime}$ CCCCAGGGATCGTTATGAGC-3' (reverse); primers \#2: $5^{\prime}$ CCGGCGAGATCTCTTTTATT- $3^{\prime}$ (forward) and $5^{\prime}$-GTCAGTTAATGAAGTGTGCCA-3' (reverse); and ChIP-qPCR primers for CDH1 promoter: 5'-GGCCGGCAGGTGAACCCTCA-3' (forward) and $5^{\prime}$-GGGCTGGAGTCTGAACTGA-3' (reverse).

\section{Luciferase assay}

For luciferase assay, MDA-MB-231 was transfected using an siRNA against $Z E B 1$ or control siRNA. Cells were treated each day during 96 hours. After 48 hours of siRNA treatment, MDA-MB-231 was infected with four different lentiviral GLuc-ON Promoter Reporter Plasmids pEZX-LvPG04 (GeneCopoeia). Two POLQ promoter constructs, a $C D H 1$ promoter (GeneCopoeia; catalog no. HPRM45458LvPG04) and a negative promoter (GeneCopoeia; catalog no. NEGLvPG04) as a control, were independently transduced. The POLQ promoter $-691 \mathrm{bp}$ was generated from the $P O L Q$ promoter $-1,280 \mathrm{bp}$ (GeneCopoeia; catalog no. HPRM54321-LvPG04-01) digested by EcoR1 and Spe1 restriction enzymes and reconstituted (New England Biolabs). pEZX-LvPG04 contains the Gaussia luciferase reporter gene under the control of the indicated promoter, and SEAP as an internal

Table 1. Primer and probe list for $\mathrm{qPCR}$.

\begin{tabular}{|c|c|c|c|}
\hline Primers & DNA probe & Forward & Reverse \\
\hline GAPDH & 60 & $5^{\prime}$-AGCCACATCGCTCAGACAC-3' & $5^{\prime}$-GCCCAATACGACCAAATCC- $3^{\prime}$ \\
\hline LIG3 & 7 & 5'-GCTGGCCACAAAGTCTTCTC-3' & $5^{\prime}$-CCAGTGAAGATGTCCAGCAA-3' \\
\hline PARPI & 22 & 5'-TCTTTGATGTGGAAAGTATGAAGAA-3' & $5^{\prime}$-GGCATCTTCTGAAGGTCGAT-5' \\
\hline$P O L Q$ & 40 & 5'-GATTGAGCCAGAGTCTGTTGG-3' & 5'-TCCATAAATGATCCCATAGCTT-3' \\
\hline$U B B$ & 39 & 5'-AGGATCCTGGTATCCGCTAAC-3' & 5'-TCACATTTTCGATGGTGTCACT-3' \\
\hline ZEB1 & 57 & $5^{\prime}$-AACTGCTGGGAGGATGACAC-3' & 5'-TCCTGCTTCATCTGCCTGA-3' \\
\hline
\end{tabular}


control. Forty-eight hours after infection, cells were seeded onto 96well plates $(20,000$ cells/well). After 48 hours, the supernatants were collected to reveal the luciferase signal using the Secrete-Pair Dual Luminescence Assay Kit (GeneCopoeia, TEBU; catalog no. LF032), according to the manufacturer's recommendations. Changes in transcription activity of $P O L Q$ promoter were normalized with respect to the corresponding negative control samples. $\mathrm{CDH} 1$ promoter and negative promoter were used as controls.

\section{Hypoxanthine-guanine phosphoribosyltransferase gene mutation assay}

Cells were infected with the indicated HPRT1 sgRNA CRISPR/Cas9 All-in-One Constructs (Applied Biological Materials; catalog no. K0986605) and cultured for an additional 7 days (cells were passaged twice in this period) with Puromycin (InvivoGen; catalog no. ant-pr-1) for selection. After 7 days, cells were trypsinized, counted, and seeded at low density (500 cells for untreated conditions and 3,000 cells for 6-TG-treated conditions). For each sample, seven plates were seeded: three were left untreated to determine the cloning efficiency ( $-6 \mathrm{TG}$ condition), whereas $5 \mu \mathrm{g} / \mathrm{mL}$ 6-TG (Sigma-Aldrich; catalog no. A4882) was added to the four other plates to select hypoxanthineguanine phosphoribosyltransferase (HPRT)-deficient cells. Two weeks after the addition of 6-TG, plates were washed with PBS $1 \times$ and stained with solution containing $50 \%$ ethanol, $5 \%$ acetic acid, and $0.5 \%$ brilliant blue R (Sigma-Aldrich; catalog no. B7920). Surviving colonies were scored, and the HPRT mutation frequency was calculated as follows:

Mutation frequency $=\frac{\text { Number of } 6-\text { TG resistant clones }}{\text { Number of cells plated } \times \text { cloning efficiency }}$

$\left(\right.$ Cloning efficiency $\left.=\frac{\text { Number of survived clones on untreated plates }}{\text { Number of cells plated }}\right)$

\section{Micronuclei}

Cells were plated onto removable Chamber Slide (IBIDI; catalog no. 81201 ) and treated for 36 hours for MDA-MB-231 or 72 hours for BT20 and HCC70 with cytochalasin B in the culture media at a final concentration of $3 \mu \mathrm{g} / \mathrm{mL}$. When the majority of cells were binucleated, they were fixed in $4 \%$ formaldehyde in $1 \times$ PBS and $1 \%$ BSA (Sigma-Aldrich; catalog no. A8412) for 20 minutes at room temperature. Cells were stained with phalloidin-TRITC and Hoechst. Finally, the silicone chambers were removed and the slides were mounted with Fluoromount Aqueous Mounting Medium (Sigma-Aldrich; catalog no. F4680-25ML). A minimum of 200 binucleated cells was counted per condition.

\section{Statistical analysis}

Statistical analysis and graphs were performed using GraphPad Prism 6.0 (RRID:SCR_002798). Data are expressed as mean \pm SEM of at least three independent experiments and were analyzed using unpaired two-tailed Student $t$ tests with a Welch correction. Significance is represented with asterisks; $P<0.05$ was considered to be significant. ${ }^{*}, P<0.05$; $^{* *}, P<0.01$; $^{* *}, P<0.001$; $^{* * * *}, P<0.0001$.

\section{Results}

$P O L Q$ expression according to genomic instability in breast tumors

To explore the importance of TMEJ in breast tumorigenesis and determine its impact on cancer genome stability, we first evaluated the expression of POLQ, PARP1, and LIG3 in primary breast tumors according to their genomic landscape. We analyzed the FGA, as a consequence of episodes of CIN accumulation over the course of tumor development and progression, in 3,083 primary breast cancers from combined databases, namely TCGA-BRCA and the METABRIC. We observed that the mRNA expression of POLQ was significantly positively correlated with FGA (Fig. 1A), whereas the correlation with PARP1 and LIG3 expression was less significant (Supplementary Fig. S1A). These results highlighted POL $\theta$ as a putative marker of CIN. We then assessed the variation in POLQ, PARP1, and LIG3 expression in primary tumors with distinct genomic landscapes by comparing their abundance at the transcript level in 10 IntClust from a molecular classification of breast cancers based on genomic and transcriptomic analyses (28). The IntClust10, mostly characterized as the high-genomic instability subgroup (Fig. 1B), was greatly enriched in tumors expressing high levels of $P O L Q$ (Fig. 1C). Conversely, the expression of $P O L Q$ was reduced in IntClust 4 and IntClust3 breast tumors, characterized by a paucity of FGA, and IntClust 4 being termed the copy-number alterations (CNA)-devoid subgroup $(28,40)$.

In agreement with previous observations, we found that $52 \%$ of all claudin-low tumors belonged to the genomically stable IntCluster4 (24), while $72 \%$ of basal-like tumors were mostly found in IntClust10, characterized by a large number of genomic aberrations (Fig. 1D). We have shown previously $(24,31)$, and confirmed here with additional data, that the IntClust 4 was enriched in TNBCs with higher levels of $Z E B 1$ expression (Fig. 1E; Supplementary Fig. S1B). Although POLQ, PARP1, and LIG3 expression within the subtypes displayed similar trends (Supplementary Fig. S1B), POLQ showed the most significant differences according to subtype. Having shown that these TMEJ actors were all underexpressed in the $\mathrm{CIN}^{\text {low }}$ IntClust with high $Z E B 1$ expression, we then examined whether their regulation was coordinated. To address this, we first analyzed the steady-state levels of ZEB1, POL $\theta$, PARP1, and DNA ligase III $\alpha$ (LIG3) proteins in four basal-like and four claudin-low TNBC cell lines. Immunoblot analyses revealed low level of POL $\theta$ protein in most of the four claudin-low ZEB1-expressing cell lines compared with basal-like cells that do not express ZEB1 (Fig. 1F). No such correlation was seen for PARP1 or LIG3 (Fig. 1F). Compared with immortalized human mammary epithelial cell (HMEC-hTERT), POL $\theta$ protein appeared mostly increased in none-ZEB1-expressing cells (Supplementary Fig. S1C). Previous studies reported variable levels of $P O L Q$ expression in breast cancer cell lines (41), but the putative connection with ZEB1 was not explored. In addition, we show, using qRT-PCR, that ZEB1 expression was elevated in claudin-low cell lines, whereas $P O L Q$ was largely poorly expressed in most of the basal-like cells (Supplementary Fig. S1D). No major variation was observed at the mRNA level for PARP1 and LIG3 in the tested cell lines, as shown previously for the proteins. Taken together, these data indicate that $P O L Q$ expression is lower in ZEB1-expressing tumors and cancer cell lines.

\section{ZEB1-positive tumors are usually POLQ negative}

We next performed cooccurrence analyses for gene expression across a set of 530 primary TNBCs from the combined TCGA and METABRIC databases. Statistical analysis for mutual exclusivity using the OR calculation revealed a significant likelihood OR of $0.072(P=$ $0.005574)$, which implies that the changes found in the expression of these two genes were mostly mutually exclusive (Fig. 2A). Owing to the lack of available POL $\theta$ antibodies for IHC detection, we then used RNAscope to monitor POLQ expression in 10 TNBCs characterized previously for ZEB1 expression (24), by in situ IHC staining (Fig. 2B). $P O L Q$ expression was low in seven, high in two, and intermediate in 

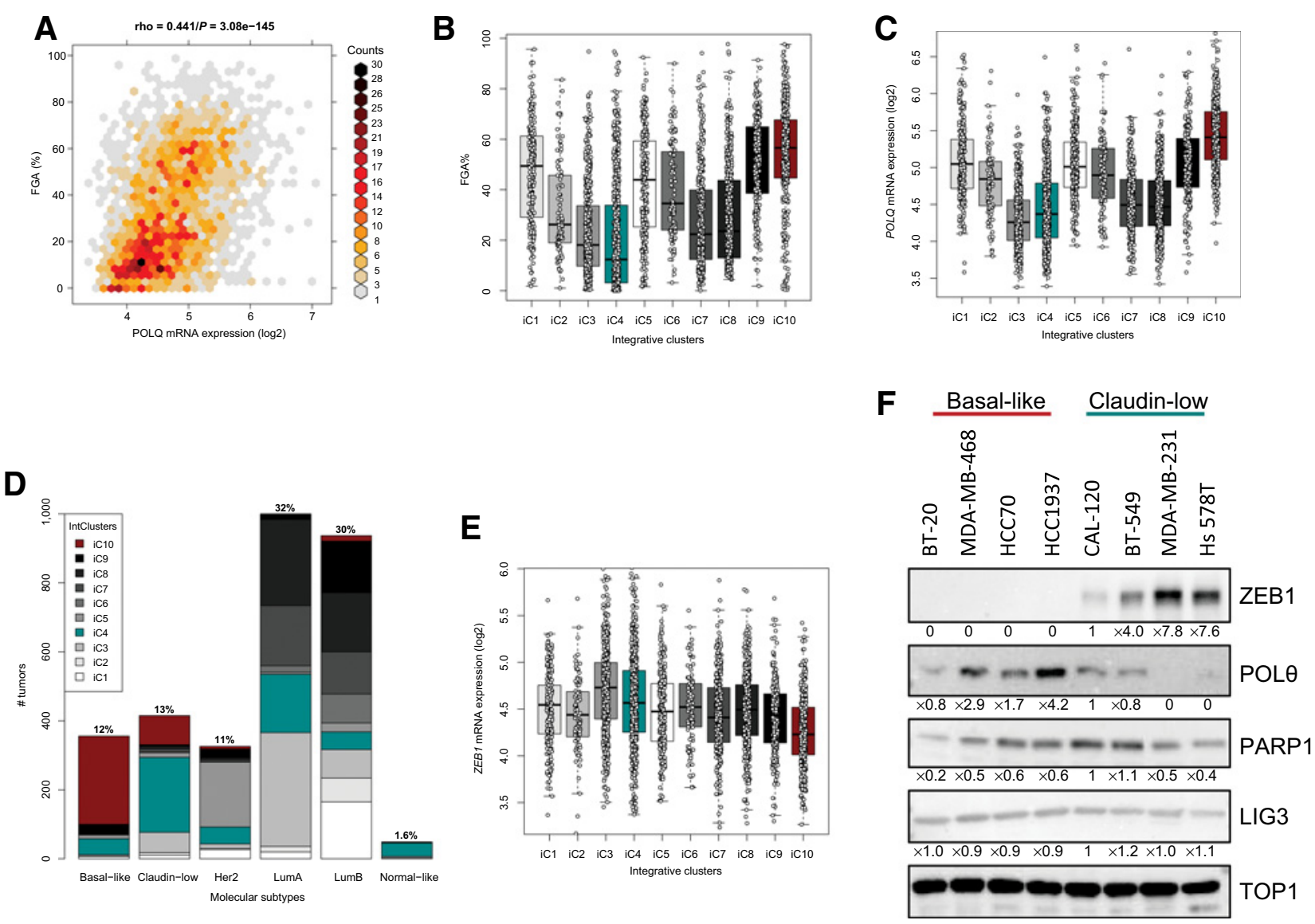

Figure 1.

$P O L Q$ expression according to genomic instability in breast tumors. A, Pearson correlation between the FGA and POLQ mRNA expression in 3,083 breast tumors for the combined TCGA and METABRIC datasets. The color code indicates the number of samples (counts) corresponding to each color. B, C, and E, FGA (B), $P O L Q$ mRNA expression (C), and ZEB1 mRNA expression (E) in subgroups (IntClust, iC) of breast tumors. Analyses were performed for the combined METABRIC and TCGA breast cancer datasets. Results are expressed as $\log _{2}$ of mRNA expression. Significance was determined using the Mann-Whitney $U$ test. Central line, median; points, all data points. D, Distribution of IntClust among breast molecular subtypes (PAM50 + claudin-low). F, Immunoblot showing the protein levels of ZEB1, POLA, PARP1, and DNA ligase III (LIG3) in TNBC cell lines normalized to CAL-120. DNA topoisomerase I (TOP1) served as a loading control. Noted at the bottom are the mean levels of protein (normalized to correct for loading differences using the TOP1 as control) for the representative Western blot analysis presented; $n=4$ independent experiments.

one of the 10 ZEB1-expressing tumors (Fig. 2C; Supplementary Fig. S2A). Conversely, POLQ expression was high in 16 of the 22 non-ZEB1-expressing TNBCs. It is worth mentioning that a single tumor sample displayed a ZEB1-positive staining in one part of the tumor with a $P O L Q$ negative one, whereas the opposite was observed in another part (Supplementary Fig. S2B), therefore, highlighting the mutual exclusivity of $P O L Q$ and ZEB1 expression in tumor presenting intratumor heterogeneity. Altogether, these results revealed an overall mutual exclusivity of ZEB1 and POLQ expression.

\section{ZEB1 regulates $P O L Q$ expression}

Given the lower expression of $P O L Q$ in ZEB1-expressing claudinlow cancer cell lines, compared with basal-like cell lines, we hypothesized that the ZEB1 EMT-inducing transcription factor (EMT-TF) may regulate $P O L Q$ expression. To address this, we first engineered $\mathrm{ZEB1}^{-1-}$ cell lines using the CRISPR-Cas9 gene editing technology in the claudin-low MDA-MB-231 cell line (Supplementary Fig. S3A) and demonstrated that ZEB1 depletion resulted in a significant increase in
POLO protein (Fig. 3A) and mRNA levels (Supplementary Fig. S3B). Second, a depletion of ZEB1 using an siRNA approach (Fig. 3B) over 4 days resulted in an increase in POL $\theta$ protein and mRNA levels in this model (Supplementary Fig. S3C). Equivalent results were obtained by using two ZEB1 siRNAs for depletion in the MDA-MB-231, BT-549, and SUM159 cell lines (Supplementary Fig. S3D). Conversely, overexpression of ZEB1 in the BT-20 basal-like cell line decreased the POL $\theta$ protein (Fig. 3C) and mRNA levels (Supplementary Fig. S3E), as well as in HCC70 cells (Supplementary Fig. S3F). Collectively, these findings suggest that $P O L Q$ expression is negatively regulated by ZEB1 in claudin-low cell lines. To explore whether this repression involved a direct interaction with the POLQ promoter, we analyzed the JASPAR database (http://jaspar.binf.ku.dk). We identified three putative ZEB1 binding sites within this region between positions $-1279 /-1269$, $-1191 /-1181$, and $-712 /-704$ upstream of the transcription start site, conforming with the optimal recognition sequence of ZEB1 (CACCTG). Two putative E-boxes, binding sites for all of the EMT-TFs and other transcription factors (CANNTG), were identified 
A

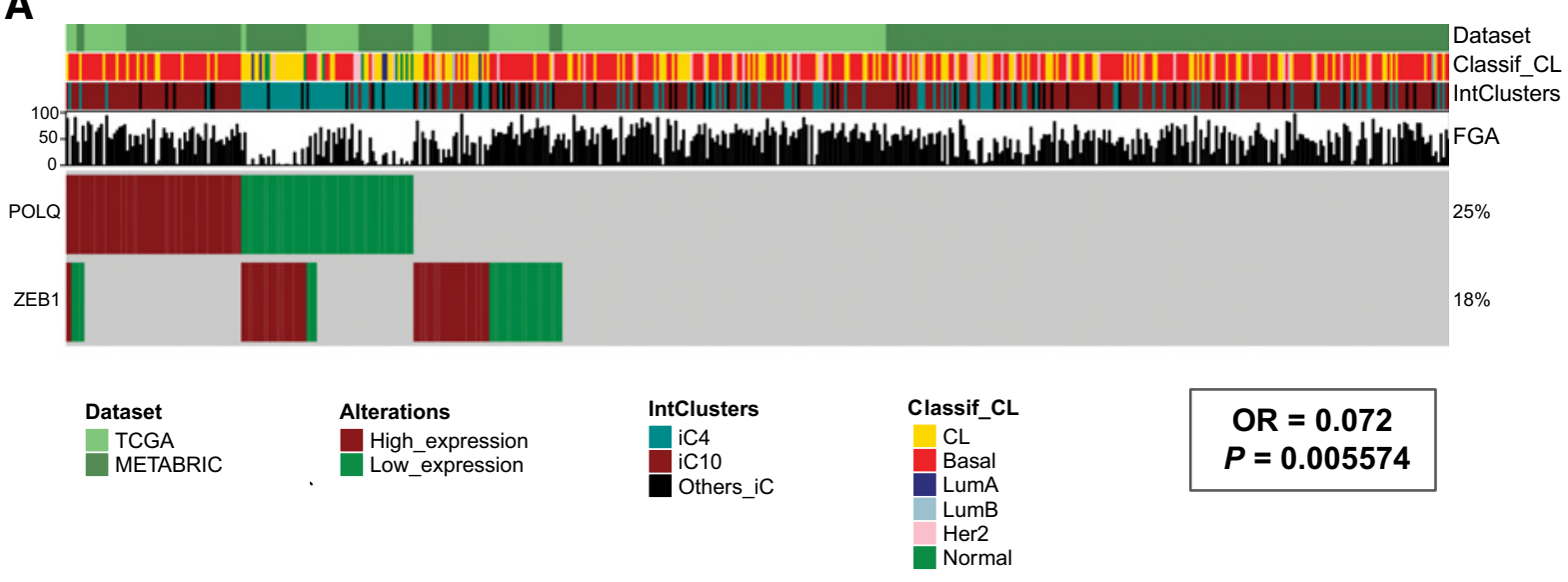

B
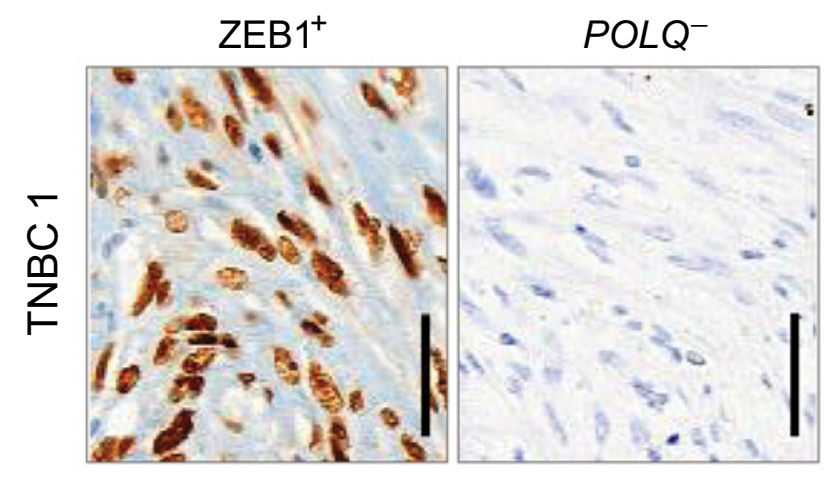

C
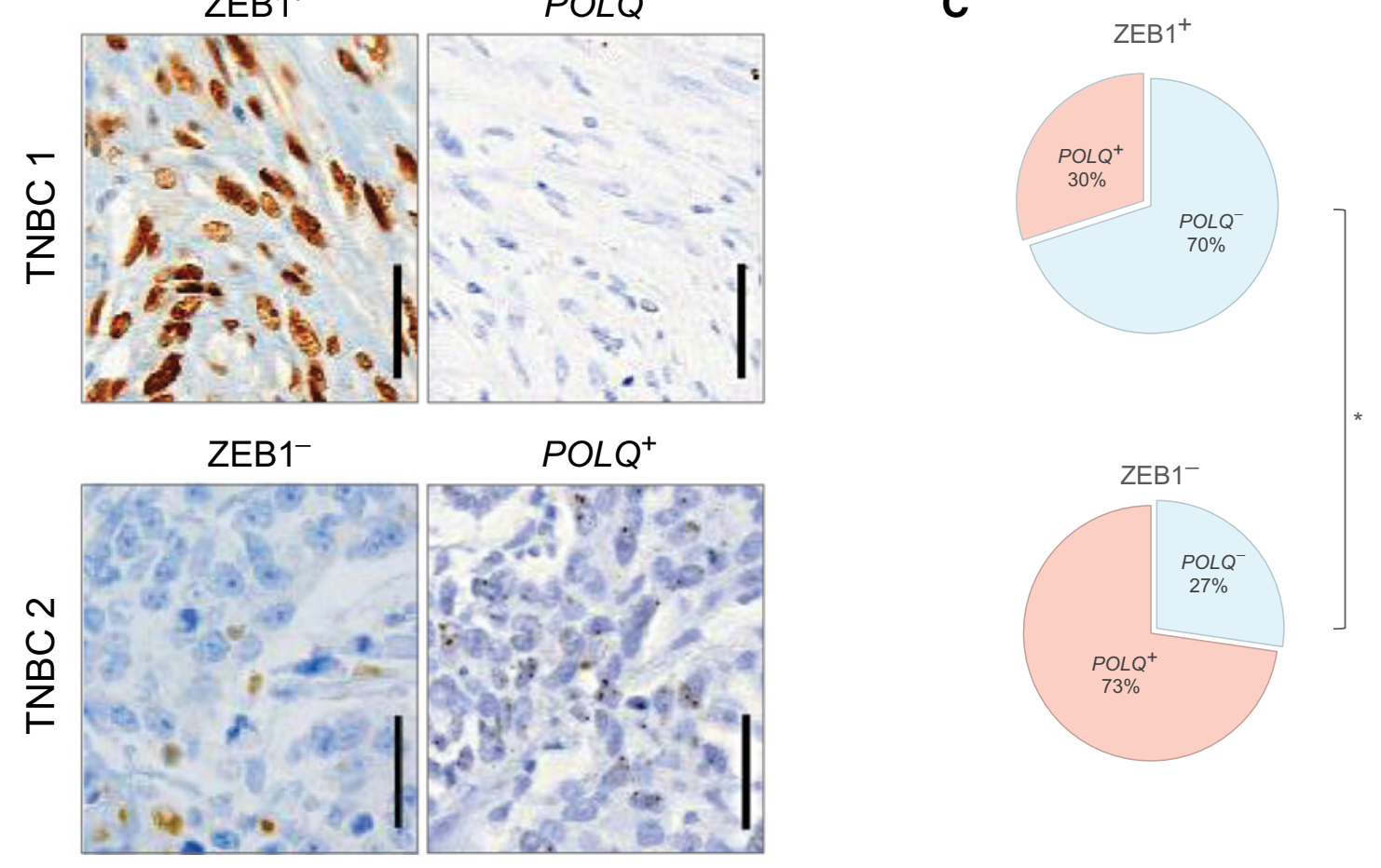

Figure 2.

ZEB1-positive tumors are usually POLQ negative. A, OncoPrint of high and low expression for POLQ and ZEB1 genes. The OncoPrint provides an overview of genomic alterations (legend) in particular genes (rows) affecting particular individual samples (columns). Gene expression of $P O L Q$ and ZEBI is mutually exclusive in TNBC as evidenced by the OR. Molecular breast cancer subtype, FGA, as well as IntClust for each sample are depicted. $N=3,083$. B, Representative IHC of two serial sections of a negative and a positive case of TNBC for ZEB1 (brown) and RNAscope for POLQ (brown dots) showing mutual exclusion in cancer cells. Scale bars, $40 \mu \mathrm{m}$ for high magnification pictures. C, Distribution of $P O L Q$ expression staining in 10 ZEB1-positive and 22 ZEB1-negative TNBCs. ${ }^{*}, P<0.05$; unpaired two-tailed Student $t$ test with Welch correction.

at positions $-1076 /-1071$ and $-662 /-654$ (Fig. 3D; Supplementary Fig. $\mathrm{S} 3 \mathrm{G}$ and $\mathrm{S} 3 \mathrm{H})$. We then performed ChIP analyses and demonstrated that ZEB1 was able to bind to the $P O L Q$ promoter (Fig. 3E) compared with ZEB1 depleted and IgG controls, as demonstrated previously for the $C D H 1$ promoter used as a positive control (42). To determine the significance of the ZEB1 binding to the promoter of $P O L Q$, the promoter region of $P O L Q$ was cloned into luciferaseexpressing plasmids (Supplementary Fig. S3I). The model was then validated by analyzing $\mathrm{CDH} 1 \mathrm{mRNA}$ levels after knocking down ZEB1 expression in the MDA-MB-231 breast cancer cell line using siRNA (Supplementary Fig. S3J). ZEB1 depletion resulted in a significant increase in $P O L Q$ promoter activity for the -1280 bp construct (Supplementary Fig. S3K), suggesting a downregulation of the POLQ promoter by ZEB1. We then generated a -691 -bp construct lacking the ZEB1 boxes of the promoter. We observed significantly less activity of the $P O L Q$ promoter. The stimulation following ZEB1 depletion was 
A

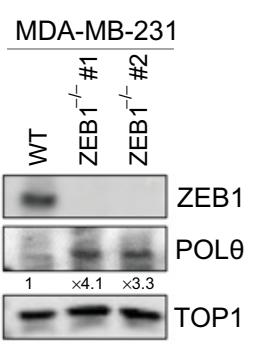

B

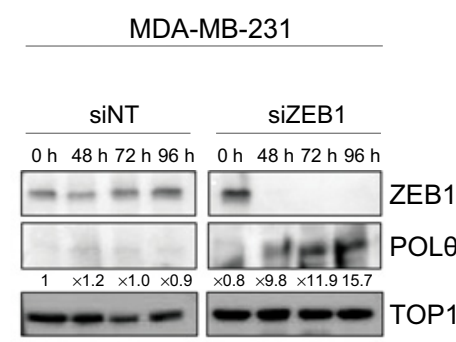

C

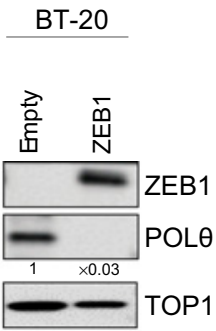

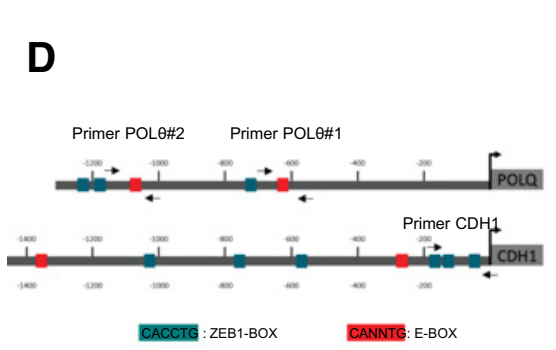

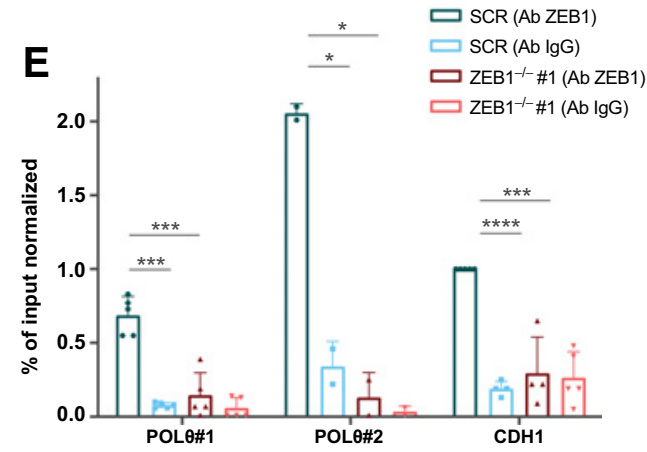

Figure 3.

ZEB1 regulates POLQ expression. A, Immunoblot showing the protein levels of ZEB1 and POL $\theta$ in stable depletion of ZEB1 in MDA-MB-231 cells (ZEB1 ${ }^{-/-} \# 1$ or $\# 2) ; n=4$ independent experiments. B, Kinetics of POL $\theta$ protein level after transient knockdown with sizEB1\#1. Western blot analysis of noted cell types assessing expression of endogenous ZEB1 and POL $\theta$ proteins. C, Immunoblot showing the protein levels of ZEB1 and POL $\theta$ in stable overexpression of ZEB1 in BT-20 cells; $n=4$ independent experiments. DNA topoisomerase I (TOP1) served as a loading control. Noted at the bottom are the mean levels of protein (normalized to correct for loading differences using the TOP1 as control) for the representative Western blot presented. $n=4$ independent experiments. D, Schematic representation of the promoter region of human POLQ (top) and CDH1 (bottom) genes on chromosomes $3 q 13.33$ and 16q22.1, respectively. The potential ZEB1 boxes (ZEB1-box CACCTG in blue) and E-box (CANNTG in red) and the regions amplified after ChIP (arrows represent primer pairs) are depicted. Numbers indicate positions in bp on chromosomal DNA relative to the transcription start site $(+1)$. E, GPCR analysis after ChIP for endogenously expressed ZEB1 in ZEB1-depleted (ZEB1 ${ }^{-/-} \# 1$ ) or control (SCR) MDA-MB-231 cells, using ZEB1 antibody (Ab ZEB1) or control IgG (Ab IgG). CDH1 was used as positive control; $n=3$ independent experiments. Mean \pm SEM. ${ }^{*}, P<0.05 ;{ }^{* * *}, P<0.001 ;{ }^{* * *}, P<0.0001$; unpaired two-tailed Student $t$ test with Welch correction.

no longer detected, suggesting a role for the $-1280 /-691 P O L Q$ promoter in ZEB1 repression. Together, these results indicate that $P O L Q$ is a direct transcriptional target of ZEB1 and that ZEB1 represses $P O L Q$ expression.

\section{ZEB1 modulates TMEJ}

We next focused on the functional interaction between ZEB1 and $P O L Q$ to mechanistically unravel the potential, as yet unreported, role of ZEB1 in TMEJ regulation. We directly assessed the intrinsic mutagenic TMEJ activity by evaluating the repair of a single genomic DSB in ZEB1-expressing compared with nonexpressing tumor cells. To this end, we used a selection-based assay that captures mutagenic end-joining described previously (43), in MDA-MB-231 claudin-low cell lines and in their ZEB1-depleted counterparts. Briefly, a unique site-specific DSB in the selectable HPRT marker gene was induced by CRISPR-Cas9 using a guided RNA directed against HPRT exon 2 (or exon 1 and 3 to evaluate the impact of the DSB location). HPRT enzymatic activity converts 6-TG drug into toxic nucleotides inducing cell death. Mutagenic repair of the targeted DSB in the HPRT gene leads to loss of HPRT protein expression or expression of an inactive HPRT protein, and renders cells resistant to 6-TG treatment (Fig. 4A). Thus, the frequency of HPRT mutations reflects the efficacy of mutagenic DSB repair and TMEJ activity. Colony-forming assay analysis revealed a significant increase in mutation frequency in
ZEB1-depleted cells (Fig. 4B), reflecting the increased level of POL $\theta$ (Supplementary Fig. S4A). As expected, no change in mutational frequency was observed following POLQ knockdown (Fig. 4B) because ZEB1-expressing cells displayed very low levels of POLO. Yet, combined siZEB1/siPOLQ rescued the increase in HPRT mutation observed in siZEB1, confirming that the HPRT assay mirrors TMEJ activity (43). A significant increase in the mutational frequency in ZEB1-depleted cells compared with wild-type cells for the DSB induced both in exon 1 and 3 (Supplementary Fig. S4B-S4E) was observed, suggesting no location effect of the single DSB generated. These data led us to propose that mutagenic repair of the DSB was alleviated in claudin-low cells because of the reduction in POL $\theta$ steadystate protein levels by ZEB1. Next, we generated an MDA-MB-231 model ectopically overexpressing POL $\theta$ and observed that it recapitulated the effects of ZEB1 depletion, that is, a significant increase in the mutational frequency (Fig. 4C; Supplementary Fig. S4F), further validating our hypothesis.

\section{ZEB1-mediated micronuclei formation by POLQ repression}

It has been reported previously in mouse models that either Polq mutation (44) or Polq depletion (45) leads to increased numbers of spontaneous micronuclei. Various molecular mechanisms contribute to micronuclei formation, including impaired DNA repair response and persistence of DSBs during mitosis associated with a defect in 

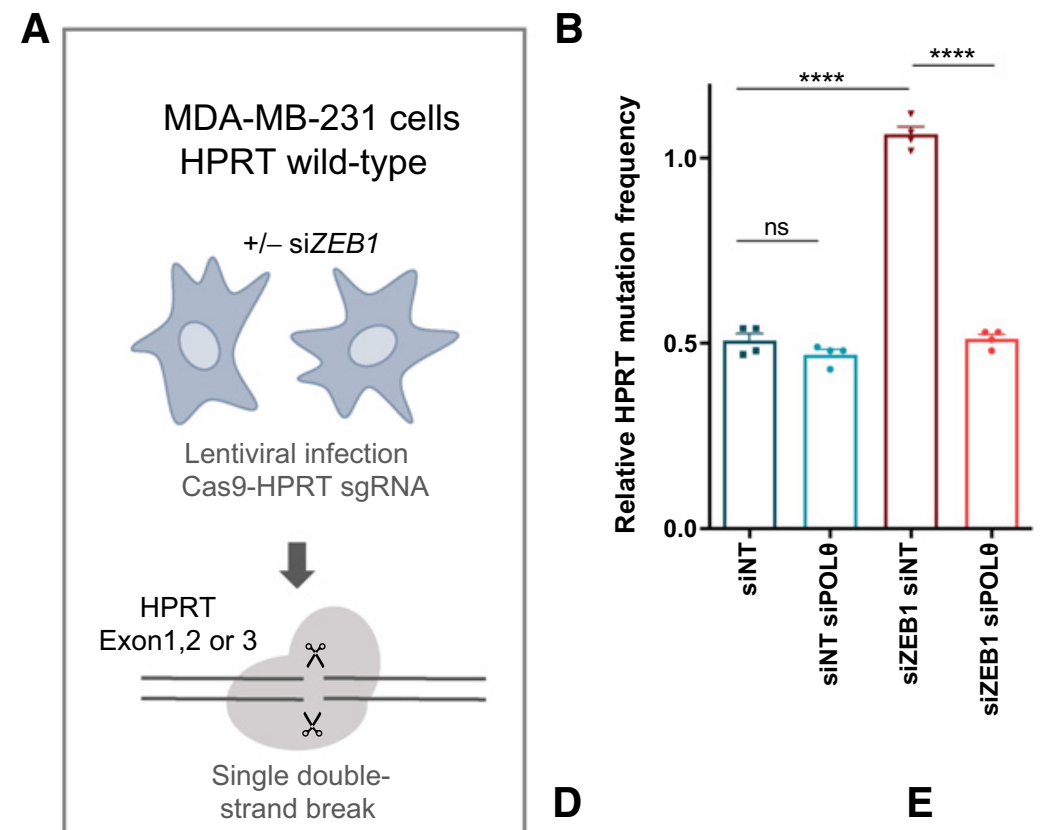

D

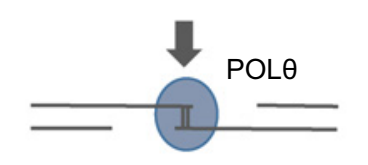

Error-prone TMEJ
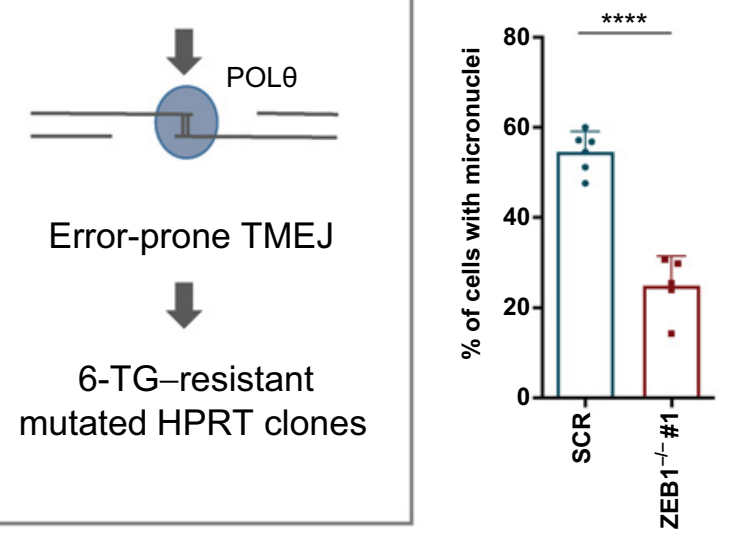

E

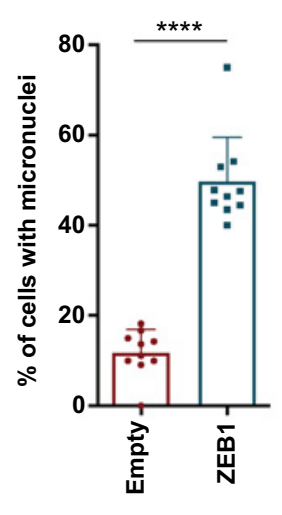

C

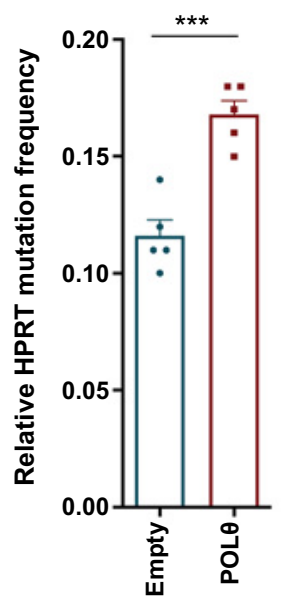

$\mathbf{F}$

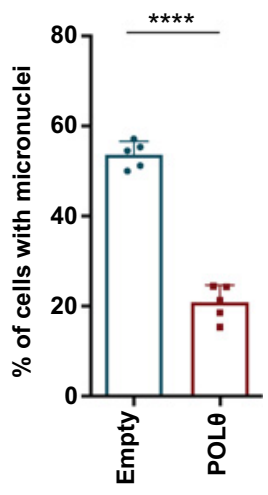

Figure 4.

ZEB1 modulates TMEJ and ZEB1 mediates micronuclei formation by POLQ repression. A, Schematic outline of HPRT mutagenic test. B, Relative HPRT mutation frequency after the indicated siRNA treatment with Cas9-WT targeting exon 2. C, MDA-MB-231 cells with forced POLQ expression. The data shown represent the mean \pm SEM; $n=3$ independent experiments; and are expressed as a fraction of the mutation frequency observed in control cells expressing a nontargeted siRNA. D, Percentage of cells with micronuclei in ZEB1-depleted (ZEB1 ${ }^{-1-}$ \#1) or control (SCR) MDA-MB-231 cells. E, BT-20 cells with forced ZEB1 expression. F, MDA-MB-231 cells with forced $P O L Q$ expression stained with Hoechst after cytochalasin B treatment. Statistical significance was calculated via unpaired two-tailed Student $t$ test with Welch correction. ${ }^{* * *}, P<0.001 ;{ }^{* * *}, P<0.0001$; ns, not significant.

DNA repair pathways (46). To address the consequences of a reduction of POLQ expression by ZEB1 in breast cancer cells, we investigated whether ZEB1 was associated with elevated levels of micronuclei in our previously described models. Consistent with our hypothesis, more than $50 \%$ of ZEB1-expressing cells, characterized by low POL $\theta$ levels, presented micronuclei (Fig. 4D). Importantly, ZEB1 depletion promoted a significant decrease in the number of micronuclei (lower than 25\%), while the forced expression of ZEB1 in BT-20 resulted in a significant increase in micronuclei (Fig. 4E), as well as in HCC70 (Supplementary Fig. S4G). Moreover, the forced expression of POL $\theta$ in ZEB1-expressing cells also led to a marked decrease in their number (Fig. 4F). These data suggest that the negative regulation of POL $\theta$ by ZEB1 in claudin-low breast cancer cells contributes to micronuclei formation. Collectively, these findings argue in favor of an essential role for POL $\theta$ during the error-prone
TMEJ mechanism in preserving genomic integrity at the cost of enhancing genetic alterations.

\section{Discussion}

In conclusion, we provide evidence of the mechanism of TMEJ regulation involving the EMT-inducing transcription factor, ZEB1, and show how the interplay between ZEB1 and POLQ may impact cancer genome stability and integrity (Fig. 5).

First, we highlight a relatively generalized mutual exclusivity of $P O L Q$ and ZEB1 expressions in TNBCs. High POLQ gene expression was enriched in the most genomically rearranged breast cancer subtype, IntClust10, containing HR-deficient tumors (31), confirming the previously described dependency of HR-deficient tumors on POL $\theta$ and TMEJ repair for survival in ovarian and breast tumors $(14,15)$. 


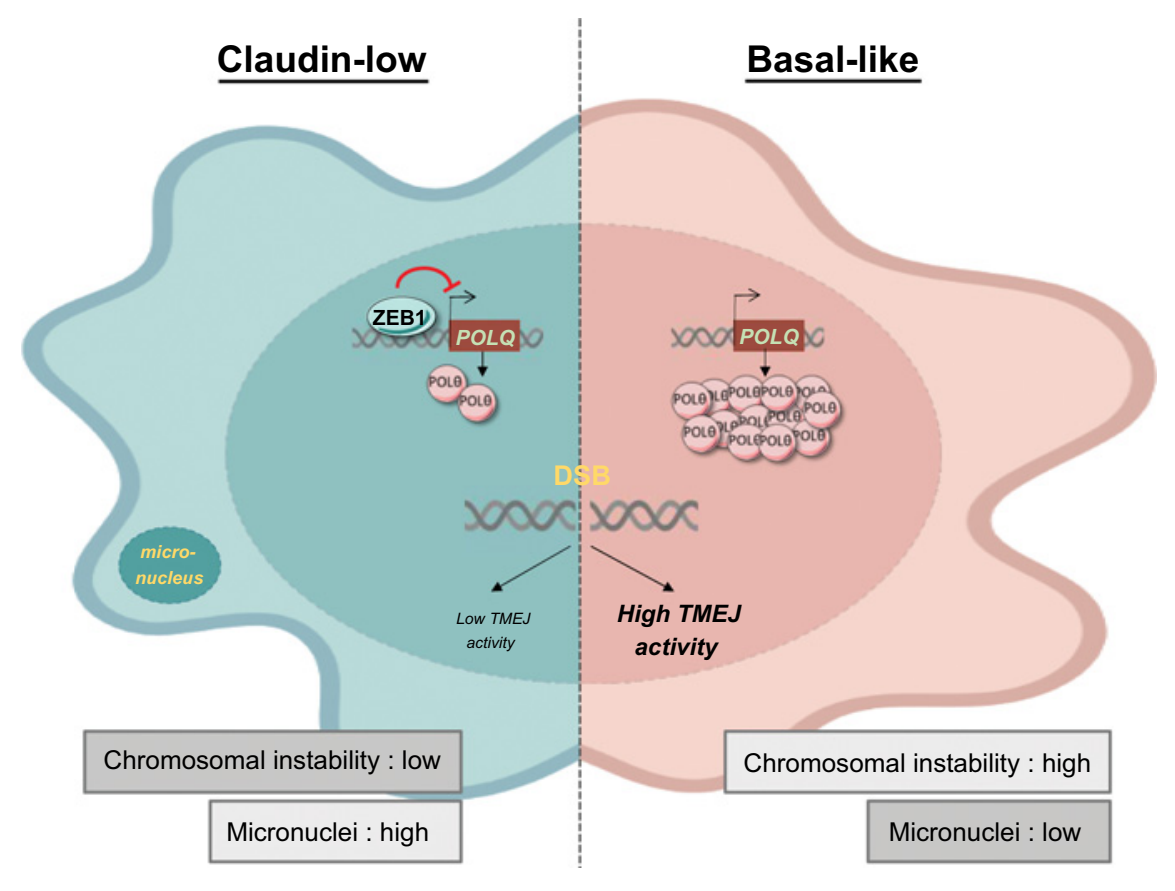

\section{Figure 5.}

Model of potential implication of TMEJ modulation by ZEB1. Among TNBC cell lines, $P O L Q$ expression was likely upregulated in the majority of basal-like tumor cell lines, especially in HR-deficient breast cancers recurrently depicted as highly genomically unstable. Nevertheless, some claudin-low cell lines exhibit low POL $\theta$ due to ZEB1 repression of $P O L Q$ gene expression. This inhibition, which we propose here modulates TMEJ activity, may participate in the low CIN observed in claudin-low tumors, but is surprisingly associated with high micronuclei in the cancer cell lines. Many mechanisms may be at the origin of micronuclei formation, but it can, however, not be excluded that low POL $\theta$ protein level, associated with low TMEJ activity, may induce residual DNA damage at the origin of micronuclei formation.
Our findings also show that some TNBCs displayed lower POLQ expression. Indeed, lower $P O L Q$ expression was observed in the IntClust 4 subgroup encompassing high-ZEB1-expressing claudinlow tumors that exhibit a paucity of genomic aberrations $(24,31)$. Our findings supported the notion that POL $\theta$ plays a role in the onset of some type of chromosomal alterations in breast tumors. Nevertheless, not all of the chromosomal alterations can be ascribed to POL $\theta$ within TMEJ. For instance, some large deletions have been shown to arise in Caenorhabditis elegans genome in the absence of POL $\theta$ (47), and articles have reported that, in c-NHEJ-deficient cells, POL $\theta$ could also protect against CIN in noncancer cells $(45,48)$. However, instability of the genome is inherent to the great majority of human cancers (1), and POL $\theta$ is largely upregulated in highly unstable human cancers, including breast (49), ovarian $(14,50)$, lung, gastric, and colorectal (51). As we have shown that POLQ expression is highly correlated with genome instability, we suggest that the low CIN instability observed in claudin-low tumors is partly due to $P O L Q$ repression by ZEB1.

Second, we confirmed that micronuclei are observed in POLQdeficient cells, and we showed that their number increased in a ZEB1-dependent manner. We proposed that increased micronuclei number occurred likely as a result of unrepaired break accumulation due to TMEJ defect. It is unclear how chromosomally unstable cancer cells cope with the presence of micronuclei. Surprisingly, in claudin-low tumors, the loss of genome integrity, that is, micronuclei increase, seems to preserve genome stability. Nevertheless, these observations are consistent with the concept that ZEB1 acts in cancer progression by protecting the genome against instability. We have previously shown that ZEB1 withstands an oncogenic activation by driving an antioxidant program, leading to a process of malignant transformation in the absence of exacerbated genomic instability (24). Similarly, it was suggested that ZEB1 is required for HR-mediated DNA damage repair and the clearance of DNA breaks (25). We report here that ZEB1 prevents the highly mutagenic TMEJ in claudin-low cells displaying micronuclei. In cancer cells, TMEJ would, therefore, be a full-fledged repair pathway, that is, a factor assisting cancer cell survival similarly to replication stress (52). As suggested previously (24), ZEB1 may foster plasticity through cell adaptability rather than genomic variation. Future approaches need to address how TMEJ thus participates in genome integrity in human cells.

Finally, to further our understanding of the biological complexity of claudin-low tumors and ultimately improve the outcomes of patients with breast cancer, our data may have clinical implications. The TMEJ downregulation that we characterized in claudin-low cells, may trigger a compensatory increase in the c-NHEJ. Although this hypothesis remains to be tested, assessment of the sensitivity of claudin-low tumors to NHEJ inhibitors may be a strategy for treating patients with claudin-low cancers.

\section{Authors' Disclosures}

No disclosures were reported.

\section{Authors' Contributions}

M.K. Prodhomme: Conceptualization, resources, data curation, formal analysis, validation, investigation, visualization, methodology, writing-original draft, writingreview and editing. R.M. Pommier: Resources, data curation, software, formal analysis, validation, writing-original draft, writing-review and editing. C. Franchet: Data curation, formal analysis, visualization, methodology, writing-original draft. F. Fauvet: Resources, data curation, formal analysis, validation. V. Bergoglio: Conceptualization, resources, validation, methodology, writing-original draft. P. Brousset: Conceptualization, resources, supervision. A.-P. Morel: Resources, supervision, funding acquisition, writing-original draft, project administration. A.-C. Brunac: Data curation, formal analysis, visualization, methodology, writingoriginal draft. M. Devouassoux-Shisheboran: Resources, data curation, formal analysis, validation. V. Petrilli: Resources, formal analysis, validation, methodology. C. Moyret-Lalle: Conceptualization, supervision, validation, writing-original draft, writing-review and editing. J.-S. Hoffmann: Conceptualization, resources, supervision, validation, writing-original draft, writing-review and editing. A. Puisieux: Conceptualization, formal analysis, supervision, funding acquisition, validation, investigation, writing-original draft, project administration, writingreview and editing. A. Tissier: Conceptualization, formal analysis, supervision, funding acquisition, validation, investigation, methodology, writing-original draft, writing-review and editing. 


\section{Acknowledgments}

The authors thank B. Manship for proofreading the article. This work was supported by funding from the Ligue contre le Cancer (EL2016.LNCC/AIP and EL2019.LNCC/AIP) and SIRIC LYriCAN (INCa-DGOS-Inserm_12563) M.K. Prodhomme was recipient of fellowships from the Ligue contre le Cancer.

\section{References}

1. Hanahan D, Weinberg RA. Hallmarks of cancer: the next generation. Cell 2011; 144:646-74.

2. Halazonetis TD, Gorgoulis VG, Bartek J. An oncogene-induced DNA damage model for cancer development. Science 2008;319:1352-5.

3. Ceccaldi R, Rondinelli B, D'Andrea AD. Repair pathway choices and consequences at the double-strand break. Trends Cell Biol 2016;26:52-64.

4. O'Driscoll M, Jeggo PA. The role of double-strand break repair-insights from human genetics. Nat Rev Genet 2006;7:45-54.

5. Scully R, Panday A, Elango R, Willis NA. DNA double-strand break repairpathway choice in somatic mammalian cells. Nat Rev Mol Cell Biol 2019;20: 698-714.

6. Seol J-H, Shim EY, Lee SE. Microhomology-mediated end joining: good, bad and ugly. Mutat Res 2018;809:81-7.

7. Schimmel J, van Schendel R, den Dunnen JT, Tijsterman M. Templated insertions: a smoking gun for polymerase theta-mediated end joining. Trends Genet 2019;35:632-44.

8. Patterson-Fortin J, D'Andrea AD. Exploiting the microhomology-mediated endjoining pathway in cancer therapy. Cancer Res 2020;80:4593-600.

9. Brambati A, Barry RM, Sfeir A. DNA polymerase theta (Pol $\theta)$-an errorprone polymerase necessary for genome stability. Curr Opin Genet Dev 2020; 60:119-26.

10. Simsek D, Jasin M. Alternative end-joining is suppressed by the canonical NHEJ component Xrcc4-ligase IV during chromosomal translocation formation. Nat Struct Mol Biol 2010;17:410-6.

11. Chiarle R, Zhang Yu, Frock RL, Lewis SM, Molinie B, Ho Yu-J, et al. Genomewide translocation sequencing reveals mechanisms of chromosome breaks and rearrangements in B cells. Cell 2011;147:107-19.

12. Hwang T, Reh S, Dunbayev Y, Zhong Yi, Takata Y, Shen J, et al. Defining the mutation signatures of DNA polymerase $\theta$ in cancer genomes. NAR Cancer 2020;2:zcaa017.

13. Carvajal-Garcia J, Cho J-E, Carvajal-Garcia P, Feng W, Wood RD Sekelsky J, et al. Mechanistic basis for microhomology identification and genome scarring by polymerase theta. Proc Natl Acad Sci U S A 2020;117:8476-85.

14. Ceccaldi R, Liu JC, Amunugama R, Hajdu I, Primack B, Petalcorin MIR, et al Homologous-recombination-deficient tumours are dependent on Pol $\theta$-mediated repair. Nature 2015;518:258-62.

15. Mateos-Gomez PA, Gong F, Nair N, Miller KM, Lazzerini-Denchi E, Sfeir A. Mammalian polymerase $\theta$ promotes alternative NHEJ and suppresses recombination. Nature 2015;518:254-7.

16. Seki M, Wood RD. DNA polymerase $\theta$ (POLQ) can extend from mismatches and from bases opposite a (6-4) photoproduct. DNA Repair 2008; 7:119-27.

17. Wood RD, Doublié S. DNA polymerase $\theta$ (POLQ), double-strand break repair, and cancer. DNA Repair 2016;44:22-32.

18. Perou CM. Molecular stratification of triple-negative breast cancers. Oncologist 2010;5:39-48.

19. Prat A, Perou CM. Deconstructing the molecular portraits of breast cancer Mol Oncol 2011;5:5-23.

20. Prat A, Parker JS, Karginova O, Fan C, Livasy C, Herschkowitz JI, et al. Phenotypic and molecular characterization of the claudin-low intrinsic subtype of breast cancer. Breast Cancer Res 2010;12:R68.

21. Puisieux A, Brabletz T, Caramel J. Oncogenic roles of EMT-inducing transcription factors. Nat Cell Biol 2014;16:488-94.

22. Wellner U, Schubert J, Burk UC, Schmalhofer O, Zhu F, Sonntag A, et al. The EMT-activator ZEB1 promotes tumorigenicity by repressing stemnessinhibiting microRNAs. Nat Cell Biol 2009;11:1487-95.

23. Morel A-P, Hinkal GW, Thomas C, Fauvet F, Courtois-Cox S, Wierinckx A, et al. EMT inducers catalyze malignant transformation of mammary epithelial cells and drive tumorigenesis towards claudin-low tumors in transgenic mice. PLos Genet 2012;8:e1002723.
The costs of publication of this article were defrayed in part by the payment of page charges. This article must therefore be hereby marked advertisement in accordance with 18 U.S.C. Section 1734 solely to indicate this fact.

Received August 2, 2020; revised October 12, 2020; accepted November 20, 2020; published first November 25, 2020.

24. Morel A-P, Ginestier C, Pommier RM, Cabaud O, Ruiz E, Wicinski J, et al. A stemness-related ZEB1-MSRB3 axis governs cellular pliancy and breast cancer genome stability. Nat Med 2017;23:568-78.

25. Zhang P, Wei Y, Wang Li, Debeb BG, Yuan Y, Zhang J, et al. ATM-mediated stabilization of ZEB1 promotes DNA damage response and radioresistance through CHK1. Nat Cell Biol 2014;16:864-75.

26. R Core Team. R: a language and environment for statistical computing. Available at: http://www.R-project.org/.

27. Gu Z, Eils R, Schlesner M. Complex heatmaps reveal patterns and correlations in multidimensional genomic data. Bioinformatics 2016;32:2847-9.

28. Curtis C, Shah SP, Chin S-F, Turashvili G, Rueda OM, Dunning MJ, et al. The genomic and transcriptomic architecture of 2,000 breast tumours reveals novel subgroups. Nature 2012;486:346-52.

29. Johnson WE, Li C, Rabinovic A. Adjusting batch effects in microarray expression data using empirical Bayes methods. Biostatistics 2007;8: $118-27$.

30. Leek JT, Johnson WE, Parker HS, Jaffe AE, Storey JD. The SVA package for removing batch effects and other unwanted variation in high-throughput experiments. Bioinformatics 2012;28:882-3.

31. Pommier RM, Sanlaville A, Tonon L, Kielbassa J, Thomas E, Ferrari A, et al. Comprehensive characterization of claudin-low breast tumors reflects the impact of the cell-of-origin on cancer evolution. Nat Commun 2020;11:3431.

32. Scrucca L, Fop M, Murphy TB, Raftery AE. mclust 5: clustering, classification and density estimation using gaussian finite mixture models. The R Journal 2016;8 289-317.

33. Gendoo DMA, Ratanasirigulchai N, Schröder MS, Paré L, Parker JS, Prat A, et al. Genefu: an R/Bioconductor package for computation of gene expression-based signatures in breast cancer. Bioinformatics 2016;32:1097-9.

34. Haibe-Kains B, Desmedt C, Loi S, Culhane AC, Bontempi G, Quackenbush J, et al. A three-gene model to robustly identify breast cancer molecular subtypes. J Natl Cancer Inst 2012;104:311-25.

35. Wirapati P, Sotiriou C, Kunkel S, Farmer P, Pradervand S, Haibe-Kains B, et al. Meta-analysis of gene expression profiles in breast cancer: toward a unified understanding of breast cancer subtyping and prognosis signatures. Breast Cancer Res 2008;10:R65.

36. Parker JS, Mullins M, Cheang MCU, Leung S, Voduc D, Vickery T, et al Supervised risk predictor of breast cancer based on intrinsic subtypes. J Clin Oncol 2009;27:1160-7.

37. Hu Z, Fan C, Oh DS, Marron Js, He X, Qaqish BF, et al. The molecular portraits of breast tumors are conserved across microarray platforms. BMC Genomics 2006;7:96.

38. Paquet ER, Hallett MT. Absolute assignment of breast cancer intrinsic molecular subtype. J Natl Cancer Inst 2015;107:357.

39. Fernandez-Vidal A, Guitton-Sert L, Cadoret J-C, Drac M, Schwob E, Baldacci $\mathrm{G}$, et al. A role for DNA polymerase $\hat{I}$ in the timing of DNA replication. Nat Commun 2014;5:4285.

40. Dawson S-JJ, Rueda OM, Aparicio S, Caldas C. A new genome-driven integrated classification of breast cancer and its implications. EMBO J 2013;32:617-28.

41. Wang Zi, Song Y, Li S, Kurian S, Xiang R, Chiba T, et al. DNA polymerase theta (POLQ) is important for repair of DNA double-strand breaks caused by fork collapse. J Biol Chem 2019;294:3909-19.

42. Cieply B, Farris J, Denvir J, Ford HL, Frisch SM. Epithelial-mesenchymal transition and tumor suppression are controlled by a reciprocal feedback loop between ZEB1 and Grainyhead-like-2. Cancer Res 2013;73:6299-309.

43. Schimmel J, Kool H, van Schendel R, Tijsterman M. Mutational signatures of non-homologous and polymerase theta-mediated end-joining in embryonic stem cells. EMBO J 2017;36:3634-49.

44. Shima N, Munroe RJ, Schimenti JC. The mouse genomic instability mutation chaos1 is an allele of POLQ that exhibits genetic interaction with atm. Mol Cell Biol 2004;24:10381-9. 
Prodhomme et al.

45. Yousefzadeh MJ, Wyatt DW, Takata K-I, Mu Y, Hensley SC, Tomida J, et al Mechanism of suppression of chromosomal instability by DNA polymerase POLQ. PLos Genet 2014;10:e1004654.

46. Ye CJ, Sharpe Z, Alemara S, Mackenzie S, Liu G, Abdallah B, et al. Micronuclei and genome chaos: changing the system inheritance. Genes 2019;10:3661. DOI 10.3390/genes10050366.

47. Koole W, van Schendel R, Karambelas AE, van Heteren JT, Okihara KL, Tijsterman M. A Polymerase theta-dependent repair pathway suppresses extensive genomic instability at endogenous G4 DNA sites. Nat Commun 2014;5:3216.

48. Wyatt DW, Feng W, Conlin MP, Yousefzadeh MJ, Roberts SA, Mieczkowski P, et al. Essential roles for polymerase $\theta$-mediated end joining in the repair of chromosome breaks. Mol Cell 2016;63:662-73.
49. Lemee F, Bergoglio V, Fernandez-Vidal A, Machado-Silva A, Pillaire M-J, Bieth A, et al. DNA polymerase up-regulation is associated with poor survival in breas cancer, perturbs DNA replication, and promotes genetic instability. Proc Nat Acad Sci U S A 2010;107:13390-5.

50. Konstantinopoulos PA, Ceccaldi R, Shapiro GI, D’Andrea AD. Homologous recombination deficiency: exploiting the fundamental vulnerability of ovarian cancer. Cancer Discov 2015;5:1137-54.

51. Kawamura K, Bahar R, Seimiya M, Chiyo M, Wada A, Okada S, et al. DNA polymerase theta is preferentially expressed in lymphoid tissues and upregulated in human cancers. Int J Cancer 2004;16:9-16.

52. Feng W, Simpson DA, Carvajal-Garcia J, Price BA, Kumar RJ, Mose LE, et al Genetic determinants of cellular addiction to DNA polymerase theta Nat Commun 2019;10:4286. 


\section{Cancer Research}

AGR American Association

The Journal of Cancer Research (1916-1930) | The American Journal of Cancer (1931-1940)

\section{EMT Transcription Factor ZEB1 Represses the Mutagenic POL $\theta$ -Mediated End-Joining Pathway in Breast Cancers}

Mélanie K. Prodhomme, Roxane M. Pommier, Camille Franchet, et al.

Cancer Res 2021;81:1595-1606. Published OnlineFirst November 25, 2020.

Updated version Access the most recent version of this article at: doi:10.1158/0008-5472.CAN-20-2626

Supplementary Access the most recent supplemental material at:

Material http://cancerres.aacrjournals.org/content/suppl/2020/11/25/0008-5472.CAN-20-2626.DC1

Cited articles This article cites 51 articles, 11 of which you can access for free at:

http://cancerres.aacrjournals.org/content/81/6/1595.full\#ref-list-1

Citing articles This article has been cited by 1 HighWire-hosted articles. Access the articles at:

http://cancerres.aacrjournals.org/content/81/6/1595.full\#related-urls

E-mail alerts Sign up to receive free email-alerts related to this article or journal.

Reprints and To order reprints of this article or to subscribe to the journal, contact the AACR Publications Department at Subscriptions pubs@aacr.org.

Permissions To request permission to re-use all or part of this article, use this link http://cancerres.aacrjournals.org/content/81/6/1595.

Click on "Request Permissions" which will take you to the Copyright Clearance Center's (CCC) Rightslink site. 